\title{
Reseñas
}

Manuel R. Rodríguez, A New Deal for the Tropics. Puerto Rico during the Depression Era 1932-1935, Princeton: Markus Wiener Publishers, 2010.

$\mathrm{E}$ propósito del libro de Manuel R. Rodríguez es examinar el contexto, funcionamiento, alcance y legado de la Puerto Rico Emergency Relief Administration (PRERA), que fue una oficina fundada por el gobierno de Estados Unidos en agosto de 1933 y permitió a esta potencia brindar alivio y trabajo a los desempleados y regular el capital de las grandes empresas en Puerto Rico en los peores momentos de la gran depresión y cuando la relación entre ambos países mostraba señas de creciente deterioro y fragilidad.

La historiografía tradicional señala que la PRERA se limitó a distribuir ayuda de emergencia. A New Deal for the Tropics plantea que, por el contrario, fue un proyecto de desarrollo complejo y ambicioso que se orientó a garantizar el bienestar de la población, transformó el papel del gobierno de Estados Unidos en la isla y modificó la relación colonial. El autor sigue la propuesta del antropólogo colombiano Arturo Escobar, quien ve el desarrollo como el discurso de dominio político y económico sobre América Latina que ha pretendido obtener la admisión del estado de subdesarrollo para facilitar las iniciativas del Primer Mundo y articular un consenso entre los presuntos beneficiarios del progreso que intenta imponerse.

Se trata de un libro extremadamente ordenado y claro, organizado en cinco capítulos, a su vez divididos en varias secciones, que se sustenta en numerosas fuentes primarias, entre otras las de la biblioteca presidencial de Franklin D. Roosevelt, así como en el cuidadoso seguimiento de La Rehabilitación, el poco conocido boletín de la PRERA.

El primer capítulo revisa cómo Puerto Rico enfrentó la crisis de 1929 y la larga depresión que la siguió. Admite que la isla se hallaba desde antes en pésima situación. Sostenida en el monocultivo del azúcar (aun cuando también producía café, tabaco y frutas y contaba con una industria del vestido), buena parte de la población era miserable y padecía un gran retraso en 
salud y educación. Las clases medias y altas también sufrían; si bien el sector profesional y burócrata ligado a la producción de azúcar había crecido, el capital ausente, la competencia externa y la inestabilidad de los mercados externos causaban dificultades. Desde luego, la crisis y la depresión agravaron los problemas económicos y las tensiones sociales amenazaron la estabilidad. La coalición gobernante de los partidos Republicano y Socialista se sintió amenazada por la relación del Partido Liberal con la nueva administración estadunidense de Franklin D. Roosevelt, en tanto que el Partido Nacionalista optaba por una política de violencia y confrontación contra el dominio de Estados Unidos.

Rodríguez señala que el New Deal o Nuevo Trato pretendió resolver las cosas mediante la extensión a Puerto Rico de la Agricultural Adjustement Administration (AAA) y la National Recovery Administration (NRA), aunque el carácter experimental de ambas coadyuvó a su fracaso y a que la situación se agravara, aun cuando favoreció el establecimiento de la PRERA, vista como la única oficina coherente y coordinada de la isla.

El segundo capítulo explica cómo las autoridades estadunidenses, con la estrecha cooperación de los sectores profesionales locales, lograron la aceptación local de los programas de la PRERA, a través del retrato de un terrible escenario y de los puertorriqueños como criaturas urgidas del progreso que sólo podrían obtener de un Estado ilustrado para superar así tanto su decadencia moral y racial, como los problemas socioeconómicos de los que ellos mismos tenían la culpa. Se pensó que si la población aceptaba la realidad y veía los "beneficios" que iba a recibir, estaría lista para ocupar su lugar en el mundo civilizado. La metrópoli lejana e indiferente mostró de tal modo súbita inquietud por sus ciudadanos en el Caribe y su intervención fue identificada con la mejoría. Tuvo además un foro para exhibir sus virtudes y responsabilidades. En todo caso, el gobierno de Washington fue presentado como una entidad omnipotente con autoridad para decidir qué era bueno o malo para la población.

Se concluye que la PRERA no sólo fue una estructura burocrática, sino un plan para cambiar la condición de la isla. Apelando a su obligación hacia Puerto Rico y sus ciudadanos, su empleo sirvió para probar que los "ideales" de la gran potencia eran capaces de establecer un modelo excepcional de desarrollo.

En el capítulo tercero se revisa cómo muchos profesionistas, intelectuales y políticos se sumaron con entusiasmo a la PRERA, a la que vieron como un proyecto para sacar adelante a la isla, aunque también para emplearse y

\section{()(1) $(3$}


crecer ellos en la burocracia. Formaban un sector con el conocimiento y/o la experiencia necesarios, descritos por los periódicos y el radio y en particular por La Rehabilitación como incorruptible, eficiente y perito, además de tolerante y paciente con el público y comprometidos con un discurso progresista y modernizador. Es importante la referencia que en este apartado se hace al papel de las mujeres en este sector.

El autor acierta al señalar que la actitud imperialista no es unilateral. Disiente de la historiografía tradicional en cuanto a que el fuerte discurso nacionalista surgido entonces, no fue sólo para condenar la relación colonial, sino sirvió a las autoridades de Estados Unidos y a los profesionistas para legitimar como cruzada de salvación a la PRERA, al igual que para probar que los puertorriqueños podían salvarse por sí mismos, con lo cual diluyó la violencia contra la presencia estadunidense y sentó las bases del Estado benefactor colonial que nacería después de la segunda guerra mundial.

Rodríguez analiza los diferentes programas de PRERA en el capítulo cuarto tanto como su impacto significativo y duradero en la vida de miles de puertorriqueños. Aborda primero el educativo y cómo, a través de las guarderías y la enseñanza para adultos, se intentó reforzar valores familiares y comunales para formar ciudadanos progresistas. En seguida revisa el programa agrícola, que se propuso combatir el monocultivo del azúcar, haciendo énfasis en la necesidad de disminuir la importación de alimentos a través de cultivos locales, la tecnificación de la economía rural, los jardines de vegetales, las granjas comunales y las cooperativas agrícolas. Por último explora la ayuda que se dio en toda la isla a la gente sin hogar, los migrantes urbanos, los adultos mayores y los desempleados; los proyectos de salud pública, vivienda, supresión de colonias pobres y construcción de barrios obreros; el impulso a las artes y a diversas actividades sociales.

En la segunda parte del capítulo el autor estudia cómo la gente vio, negoció e incorporó la PRERA en su vida diaria. Tanto el discurso del presidente Roosevelt, dirigido al rescate del "hombre común", como los efectos de la depresión favorecieron que él y su esposa fueran estimados como capaces de resolver todo problema personal, familiar, comunal y nacional. Sin embargo, subraya que a la vez que se brindaba auxilio a la población, los diversos programas imponían valores y hacían a los puertorriqueños más dependientes de un Estado interventor. El resultado fue que se generó un consenso a favor de la estadía y los beneficios ofrecidos por Estados Unidos.

El capítulo quinto acomete con el final de la PRERA y su legado. Se relata cómo, a pesar de sus ventajas, la administración no duró sino hasta

\section{()(1)(3)}


junio de 1936. Una razón fue que los fondos resultaron insuficientes para ayudar a casi dos tercios de la población; otra su casi de facto alianza con el Partido Liberal, que la coalición gobernante resintió acusándola de ser un gobierno paralelo. La ruptura tuvo lugar, pero no todo se perdió pues la elite política y profesional isleña había comprendido que el desarrollo que el progreso encarnado por la PRER A mejoraría las condiciones locales, preservando la estabilidad. Rodríguez plantea el desarrollo inmediato del llamado Plan Chardón, que diseñó Ernesto Chardón, el rector de la Universidad de Puerto Rico, y tuvo el apoyo de la coalición, y la Puerto Rico Reconstruction Administration (PRRA), anunciada por el gobierno de Roosevelt en mayo de $1935 \mathrm{y}$ que terminó sus programas entre 1939 y 1940, aunque su desaparición total no fue sino hasta 1955.

El primero era un plan semejante al de la PRERA, aunque más ambicioso. Pretendió hacer amplias reformas en la industria azucarera, con base en la compra de ingenios y la redistribución de tierras de producción, prestó atención a los bosques, el cultivo del café y el tabaco e incluyó programas de vivienda, supresión de barrios pobres, construcción de hidroeléctricas y caminos, aumento de los servicios escolares y formación de campos de entrenamiento para trabajadores. Interesante era su idea de ser puente entre Estados Unidos y los países de habla hispana y de generar una nueva imagen de la potencia imperialista en América Latina.

Por su parte, la PRRA, a la que se sumaría Chardón como director asistente, culminó varios programas de la PRERA. Uno fue la expropiación de los grandes productores de azúcar, otro la formación de cooperativas azucareras y del café. También realizó un gran plan de electrificación rural, obras públicas, escuelas, dispensarios médicos, viviendas y amplió los servicios de la Universidad de Puerto Rico.

El libro concluye acertadamente que el legado de ambas administraciones fue no sólo la experiencia que proveyeron para programas posteriores, sino el fijar las condiciones para el surgimiento de un nuevo modelo de gobierno y gobernantes en Puerto Rico, amén de que se redefinió la relación colonial, esto es, Estados Unidos dejó de ser la metrópoli distante y se convirtió en una metrópoli preocupada por todos sus ciudadanos, con responsabilidad sobre sus vidas y bienestar.

Nada más dos reclamaciones al autor de A New Deal for the Tropics. La primera: que es una pena que una obra bien sustentada y organizada como la suya describa a Puerto Rico, a la PRERA y la PRRA prácticamente aisladas en el tiempo y el espacio, esto es, que deje de lado el transcurrir de la Gran

\section{(1)(1) $\$$}


Depresión y el Nuevo Trato en Estados Unidos, a los cuales en buena medida respondían los sucesos en la isla caribeña: La segunda, que no insista en forma suficiente en que el final del segundo proyecto de Roosevelt se debió, en parte, al estallido de la segunda guerra mundial.

Ana Rosa Suárez Argüello Instituto de Investigaciones Dr. José María Luis Mora, México asuarez@mora.edu.mx 